\title{
CONTROL OF GLOBAL VARIABLES FOR IDENTICAL AND NON-IDENTICAL JOSEPHSON JUNCTIONS ARRAYS
}

\author{
Nikolay Litvinov \\ Faculty of Applied Mathematics and Control Processes, \\ Saint Petersburg State University, \\ Russia \\ st080999@student.spbu.ru, skaldfire@yandex.ru
}

Article history:

Received 27.10.2021, Accepted 25.11.2021

\begin{abstract}
In the paper a way to solve an LQ-problem for a Josephson junction array with a common $L R C$-load is proposed. The cases of identical and non-identical Josephson junctions are considered. The solution ensures phase stabilization of Josephson current in every junction. The results are obtained using computer simulation in Jupyter Notebook and MATLAB.
\end{abstract}

\section{Key words}

optimal control, Josephson junction, LQ-problem, qubit

\section{Introduction}

A Josephson junction is a possible way to construct a quantum bit (qubit) [Nielsen and Chuang, 2000]. A Josephson qubit can be presented as a non-linear resonator. There are three types of superconducting qubits that are distinguished by how the non-linear resonator is constructed, namely phase, flux and charge qubits. Phase and flux qubits are sensitive to the phase value of the Josephson current [Geller, 2006; Martinis and Osborne, 2004].

To perform quantum computations it is necessary to create a chain or an array of qubits. Dynamics of Josephson junctions arrays was studied in detail in a significant number of works, e.g., [Hens, Pal, Dana, 2015; Kuznetsov et al., 2018; Vlasov and Pikovsky, 2013]. Dynamics of identical non-linear oscillators network was studied in [Mishra A. et al., 2015].

Control of one Josephson junction was investigated in [Borisenok, 2015]. The Josephson junctions array may be presented as a multidimensional controlled system with periodic non-linearities. Dynamics of such systems was considered in [Smirnova, Proskurnikov, Utina, 2019].
In [Vlasov and Pikovsky, 2013] the authors show how the phases depend on the global variables of an electrical circuit: the phases are not stable and grow up with time.

In the current paper an approach to stabilization of the Josephson junctions array phases is suggested. It is based on solution of the optimal control problem for the Josephson junctions array in terms of global variables.

\section{Models description}

\subsection{Identical Josephson junctions array model}

The identical junctions can be described by the system of ordinary differential equations (ODE system) in dimensionless global variables [Kuznetsov et al., 2018; Vlasov and Pikovsky, 2013]:

$$
\left.\begin{array}{l}
\dot{x}_{i}=I-\sin x_{i}-\varepsilon x_{N+2} \\
\dot{x}_{N+1}=x_{N+2} \\
\dot{x}_{N+2}=I-\gamma x_{N+2}-\omega_{0}^{2} x_{N+1}- \\
-\frac{1}{N} \sum_{i=1}^{N} \sin x_{i}
\end{array}\right\}
$$

where $x_{i}$ is the phase of the $i$-th junction; $N$ is the total number of junctions; $x_{N+1}$ is the load capacitor charge; $I$ is the external current; $\varepsilon, \gamma$ and $\omega_{0}^{2}$ are dimensionless parameters of the parallel $R L C$-load. Initial conditions for ODE system are $x_{0}=(0, \ldots, 0,0.5,0)^{T}$.

An equivalent circuit for the identical Josephson junctions array with an $R L C$-load connected in parallel is presented in Fig. 1.

The numerical simulation for solution of (1) is given in Fig.2. The simulation was made for 200 junctions array, following to [Vlasov and Pikovsky, 2013].

From simulation follows that identical junctions are in synchronous state.

Also, the simulation for different initial values of phases was carried out. The initial values of phases are in range $[0 ; 10]$. Simulation results are presented in the 


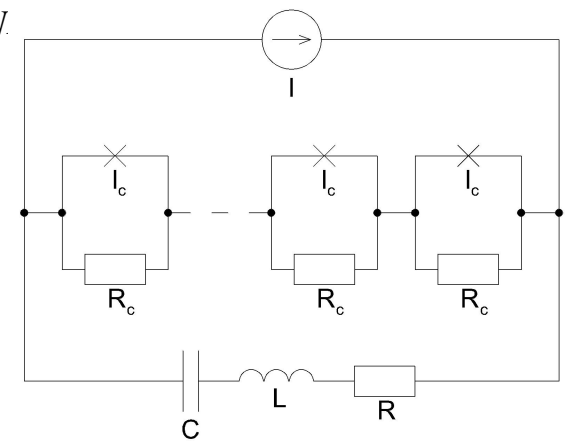

Figure 1. Equivalent circuit for the identical Josephson junction array with the common RLC load [Wiesenfeld et al.]
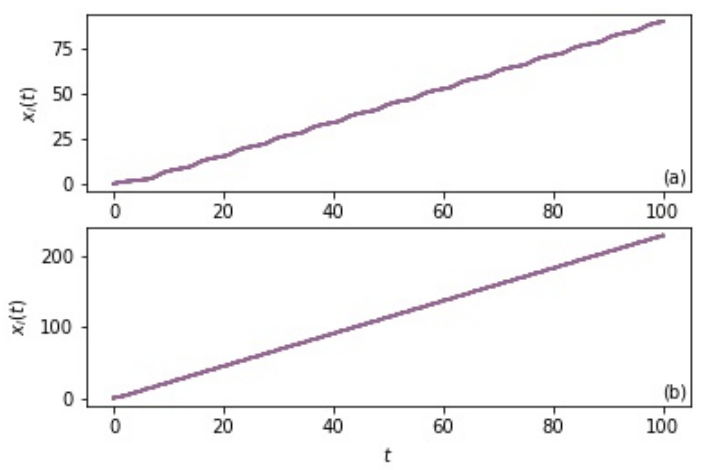

Figure 2. Identical Josephson junctions array simulation results. (a) Values of the parameters are $I=1.2, \varepsilon=0.5, \omega_{0}^{2}=1.2$, $\gamma=1$. (b) Values of the parameters are $I=2.5, \varepsilon=0.5$, $\omega_{0}^{2}=1.2, \gamma=1$.

Fig. 3. As follows from the graphs, Josephson junctions are in asynchronous state.
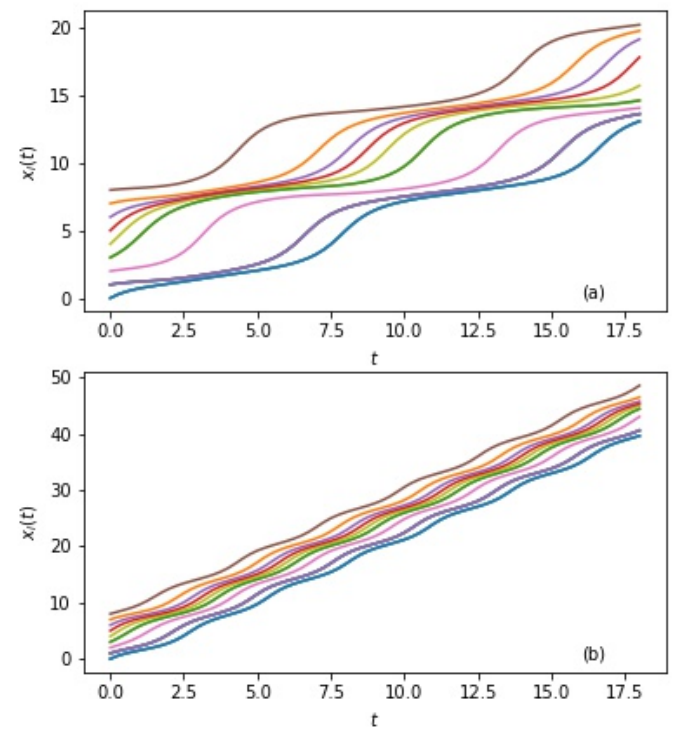

Figure 3. Identical Josephson junctions array simulation results for different initial values of the phases. 15 phases of 200 are shown. (a) Values of the parameters are $I=1.2, \varepsilon=0.5, \omega_{0}^{2}=1.2$, $\gamma=1$. (b) Values of the parameters are $I=2.5, \varepsilon=0.5$, $\omega_{0}^{2}=1.2, \gamma=1$.

where the values have the same meaning as in (1) and $\xi_{i}$ are the parameters characterizing the difference of the critical currents from the nominal value. An equivalent circuit for the non-identical Josephson junctions array with a parallel $R L C$-load is presented in Fig. 4.

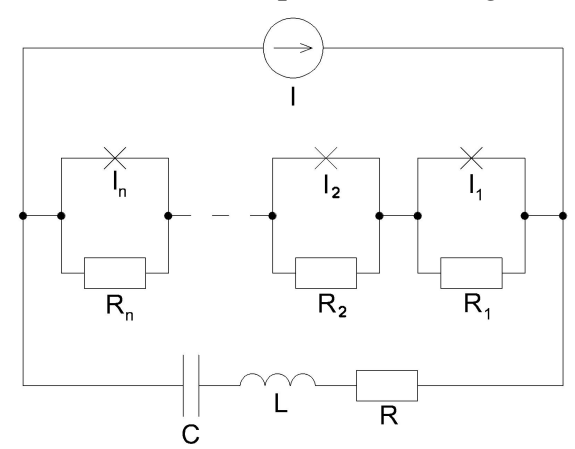

Figure 4. Equivalent circuit for the non-identical Josephson junction array with the common RLC load [Wiesenfeld et al.]
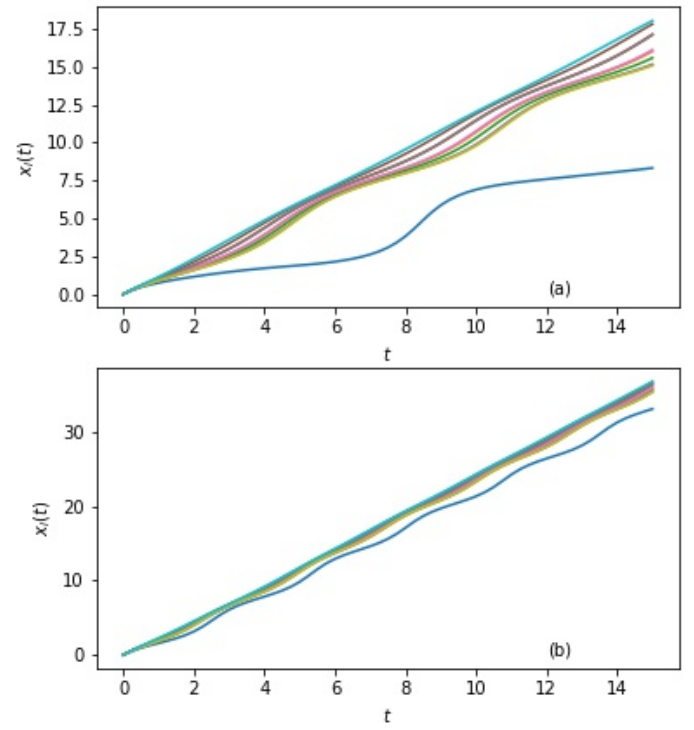

Figure 5. Non-identical Josephson junctions array simulation results. 10 phases of 200 are shown. (a) Values of the parameters are $I=1.2$, $\varepsilon=0.5, \omega_{0}^{2}=1.2, \gamma=1, \xi_{i} \in[-1 ; 1]$. (b) Values of the parameters are $I=2.5, \varepsilon=0.5, \omega_{0}^{2}=1.2, \gamma=1$, $\xi_{i} \in[-1 ; 1]$. 
The simulation of (2) is presented in the Fig. 5. The simulation was done for an array with 200 junctions, following to [Vlasov and Pikovsky, 2013].

\section{Problem statement and its solution}

Let us consider systems (1) and (2) in general form and expand them with a control function:

$$
\dot{x}=f(x)+B u+\bar{I}=F(x, u)+\bar{I},
$$

where $x=\left(x_{1}, \ldots, x_{n}\right)^{T}, x \in \mathbb{R}^{n}$,

$u=\left(u_{1}, \ldots, u_{r}\right)^{T}, u \in \mathbb{R}^{r}, r \leq n$,

$f=\left(f_{1}, \ldots, f_{n}\right)^{T}, f \in C^{\infty}\left(\mathbb{R}^{n} \times \mathbb{R}^{r} ; \mathbb{R}^{n}\right) ;$

$\bar{I}=(I, \ldots, I, 0, I)^{T}, F(0,0)=0$,

$$
B=\left(\begin{array}{cccccc}
1 & 0 & \ldots & 0 & 0 & 0 \\
0 & 1 & \ldots & 0 & 0 & 0 \\
\ldots & \ldots & \ldots & \ldots & \ldots & \\
0 & 0 & \ldots & 1 & 0 & 0 \\
0 & 0 & \ldots & 0 & 0 & 0 \\
0 & 0 & \ldots & 0 & 0 & 1
\end{array}\right)
$$

Problem. Find a pair of the functions $x(t), u(t)$, which satisfy to the system (3) and given initial conditions:

$$
x_{0}=\left(x_{0}^{1}, \ldots, x_{0}^{n}\right) .
$$

$u(t)$ is the optimal control for the linearized system (3).

Problem solution. Let us consider ODE systems (1) and (2). These systems contain significant non-linearity. Thus for the start we make Taylor expansion and keep only linear terms.

The linearized systems have general form

$$
\dot{x}=A x+B u,
$$

where $A$ is the matrix of the first approximation summands which has the form

$$
A=\left(\begin{array}{ccccc}
-\left(1+\xi_{1}\right) & \ldots & 0 & 0 & -\varepsilon \\
\ldots & \ldots & \ldots & \ldots & \ldots \\
0 & \ldots & -\left(1+\xi_{N}\right) & 0 & -\varepsilon \\
0 & \ldots & 0 & 0 & 1 \\
-\frac{1+\xi_{1}}{N} & \ldots & -\frac{1+\xi_{N}}{N} & -\omega_{0}^{2}-\gamma
\end{array}\right)
$$

The case $\xi_{i}=0, i=1, \ldots, N$ corresponds to an identical junctions array.

Test of the controllability conditions for these systems shows that

$$
\operatorname{rank} S=n,
$$

where $S=\left(B, A B, \ldots, A^{n-1} B\right), n$ is a dimension of the system.

Now we study a stationary Linear-Quadratic problem (LQ-problem) for (5), following to [Afanas'ev et al., 1989].
LQ-problem. Find a pair of the functions $x(t), u(t)$, which satisfy to the system (5) over the infinite time interval and minimize the quality criterion

$$
\begin{array}{r}
J(u)=\int_{0}^{\infty}\left[x^{T}(s) N_{2} x(s)+u^{T}(s) N_{3} u(s)\right] d s, \\
N_{3}>0, N_{2}>0,
\end{array}
$$

where matrices $N_{2}$ of dimension $[n \times n]$ and $N_{3}$ of dimension $[r \times r]$ are positively defined.

The required control function $u(t)$ that satisfies (8) is defined as

$$
u(x)=-N_{3}^{-1} B^{T} P x,
$$

where matrix $P>0$ solves the algebraic Riccati equation

$$
A^{T} P+P A+N_{2}-P B N_{3}^{-1} B^{T} P=0 .
$$

The Riccati equation (10) is solved with MATLAB Control Toolbox. We substitute the solution of the Ricatti equation $P$ to (9) to obtain the control functions. At the last step we make substitution of the obtained control functions to the initial non-linear systems.

\section{Numerical simulation results}

Simulation was done for controlled systems of the identical and non-identical Josephson junctions arrays. Initial conditions (4) for ODEs systems are $x_{0}=$ $(0, \ldots, 0,0.5,0)^{T}$. We considered two values of the external current, namely $I=1.2$ and $I=2.5$. Values of parameters $\xi_{i}$ for non-identical Josephson junctions array vary in range $[-1,1]$. Parameters of the $R L C$-load are $\varepsilon=0.5, \gamma=1, \omega_{0}^{2}=1.2$ for both cases. The parameters are taken from [Kuznetsov et al., 2018; Vlasov and Pikovsky, 2013]. We have chosen matrices $N_{2}$ and $N_{3}$ to be identity matrices of the corresponding sizes. Simulation results are presented in Fig. 6, 7.
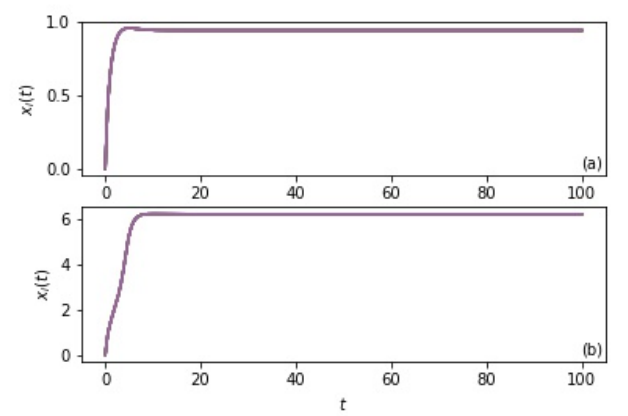

Figure 6. The simulation results for solution of the control problem for Identical Josephson junctions array. Values of the parameters are $\varepsilon=0.5, \omega_{0}^{2}=1.2, \gamma=1$. (a) $I=1.2$. (b) $I=2.5$. 

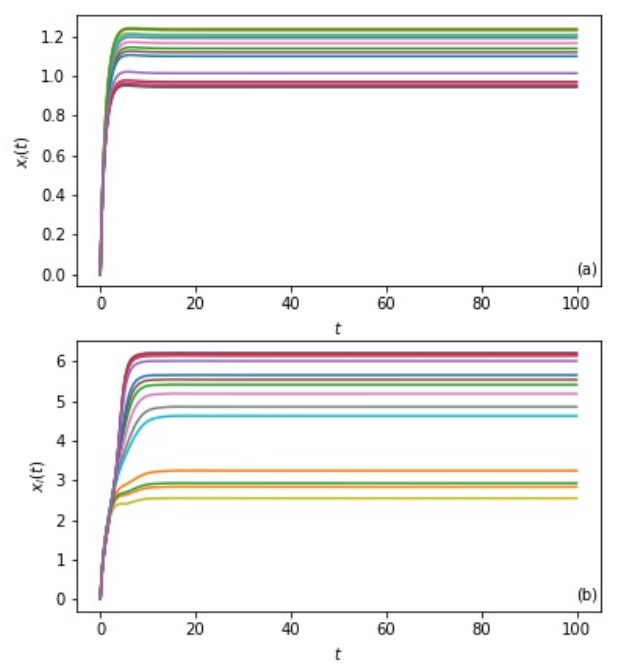

Figure 7. The simulation results for solution of the control problem for non-identical Josephson junctions array. The first 15 junctions of 200 are shown. Values of the parameters are $\varepsilon=0.5, \omega_{0}^{2}=1.2$, $\gamma=1, \xi_{i} \in[-1 ; 1]$. (a) $I=1.2$. (b) $I=2.5$.

In case of different initial phase values for identical Josephson junctions array, these values are chosen in range $[0,10]$. To determine the required external current value, numerical experiments were carried out. Results are presented in Fig. 8
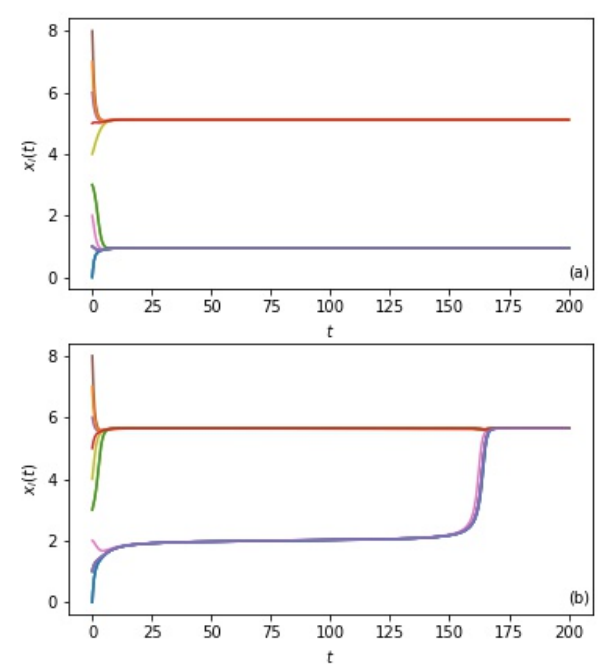

Figure 8. The simulation results for solution of the control problem for identical Josephson junctions array with different initial values of the phases. The first 15 junctions of 200 are shown. Values of the parameters are $\varepsilon=0.5, \omega_{0}^{2}=1.2, \gamma=1$. (a) $I=1.2$ (b) $I=1.738$

\section{Analysis of the simulation results}

1. The solution of the control problem ensures stabilization of the phase values for identical and nonidentical junctions arrays.

2. Dependence of the phase values from external current is detected.

3. In the case of identical junctions with different initial values of the phases the synchronization effect is detected (see Fig. 8). When external current $I<1.738$ phases tend to two different values. Phases tend to one synchronous state with an external current $I \geq 1.738$.

Numerical simulation was performed in Jupyter Notebook.

\section{Summary}

In this article an optimal control approach was applied to solving the control problem for the identical and nonidentical Josephson junctions arrays. The results of the computer simulation show that the chosen method provides stabilization of the phases for both cases. The proposed solution may be useful in quantum computer construction based on the phase qubits [Geller, 2006; Martinis and Osborne, 2004].

\section{Discussion}

In this paper problems of the possibility of measuring the whole state vector and applying of the optimal control method to a high dimension of the system are not considered. These issues will be studied in the future works.

\section{Acknowledgements}

The author is grateful to Professor Aleksandr Nikolaevich Kvitko for useful comments to the study. Also, the author is thankful to Associate Professor Alexey Eremin for discussion and correction of the article text.

\section{References}

Afanas'ev, V. N., Kolmanovskii, V. B., Nosov, V. R. (1996) Mathematical theory of control systems design. Translated and revised from the 1989 Russian original. Mathematics and its Applications, 341.

Borisenok S. (2015) Tracking with Target Attractor Feedback in Superconducting Josephson Junction, PhysCon 2015 Conference Proceedings

Geller M. R. (2006) Quantum computing with superconductors I: architectures. arXiv: quant-ph/0603224v1 24 Mar 2006.

Hens C., Pal P., Dana S.K. (2015) Bursting dynamics in a population of oscillatory and excitable Josephson junctions, Phys. Rev. E 92, 022915

Kuznetsov A.P., Sataev I.R., Sedova Yu.V. Dynamics of Three and Four Non-identical Josephson Junctions. Journal of Applied Nonlinear Dynamics, 7, 2018, No 1, pp. 105-110.

Martinis J. M., Osborne K. (2004) Superconducting qubits and the physics of Josephson junctions, arXiv:cond-mat/0402415v1 
Mishra A. et al. (2015) Chimeralike states in a network of oscillators under attractive and repulsive global coupling, Phys. Rev. E 92, 062920

Nielsen M.A., Chuang I.L., Quantum Computation and Quantum Information (Cambridge University Press, Cambridge, 2000).

Smirnova V., Proskurnikov A., Utina N. (2019) New results on cycle-slipping in pendulum-like systems, CY-
BERNETICS AND PHYSICS, Vol. 8, No. 3., pp. 167175.

Vlasov V., Pikovsky A. (2013) Synchronization of a Josephson junction array in terms of global variables, Phys. Rev. E, 88, 022908.

Wiesenfeld K., Colet P., Strogatz S.H. (1998) Frequency locking in Josephson arrays: Connection with the $\mathrm{Ku}-$ ramoto model, Phys. Rev. E 57, 1563. 